
\title{
ventilação não invasiva
}

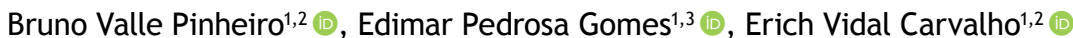

Durante a pandemia de COVID-19, muitos pacientes foram admitidos em UTIs devido à insuficiência respiratória hipoxêmica aguda (IRHA) e necessitaram de suporte ventilatório não invasivo ou invasivo. A mortalidade desses pacientes foi alta em muitas regiões. ${ }^{(1,2)}$ Pacientes com doença grave, a sobrecarga de UTIs e a falta de equipamentos, incluindo ventiladores capazes de oferecer ventilação mecânica segura e eficiente, podem ter contribuído para a mortalidade excessiva. ${ }^{(3)}$

Nesse cenário, uma força-tarefa multidisciplinar no estado do Ceará, Brasil, desenvolveu uma nova interface para a aplicação de ventilação não invasiva (VNI), que foi denominada ELMO. Os resultados de um estudo preliminar que avaliou a viabilidade, a resposta aguda e os efeitos adversos do uso de ELMO estão publicados neste número do Jornal Brasileiro de Pneumologia. ${ }^{(4)}$

O ELMO é uma interface tipo capacete que permite a aplicação de CPAP $=8-15 \mathrm{cmH}_{2} \mathrm{O}$, com $\mathrm{FIO}_{2}$ de até $100 \%$. A pressão positiva é gerada por dois fluxômetros de ar comprimido (até 30 L/min cada) e uma válvula PEEP acoplada a uma saída de ar. Um fluxo total de gás superior a $40 \mathrm{~L} / \mathrm{min}$ é suficiente para evitar a reinalação de $\mathrm{CO}_{2}$. (4) O ELMO permite aplicar VNI sem ventilador, o que é uma vantagem significativa, especialmente durante a pandemia de COVID-19, quando o número de ventiladores disponíveis era praticamente insuficiente em algumas regiões. ${ }^{(5)}$

Os resultados desse estudo preliminar mostraram que a aplicação do CPAP com ELMO (ELMOcpap) é viável. Apenas um paciente em cada dez (10\%) não tolerou o ELMOcpap e o utilizou por menos de $40 \mathrm{~min}$. A mediana do número de dias de uso do ELMOcpap foi de 2 (IIQ: 1-5 dias), com mediana de 310 min de uso diário (IIQ: 60-1.230 min). Durante o uso do ELMOcpap, os pacientes permaneceram confortáveis e não foram necessários sedativos ou analgésicos. O ELMOcpap foi associado a aumentos na $\mathrm{PaO}_{2}, \mathrm{SaO}_{2}$ e $\mathrm{PaO}_{2} / \mathrm{FiO}_{2}$, bem como à redução do escore de dispneia de Borg. A reinalação de $\mathrm{CO}_{2}$ não foi detectada. Foram observados apenas efeitos colaterais leves: tosse, boca seca, irritação nos olhos, regurgitação e desconforto cervical/axilar. ${ }^{(4)}$ A taxa de sucesso do ELMOcpap foi de $60 \%$, resultado semelhante ao encontrado em outros estudos que aplicaram VNI em pacientes com IRHA por COVID-19. ${ }^{(6)}$ Quatro pacientes falharam: um não tolerou a interface e recebeu oxigenoterapia convencional e três tiveram piora do quadro respiratório e foram intubados. Desses três pacientes, dois faleceram. ${ }^{(4)}$
A eficácia da VNI com capacete em IRHA foi previamente demonstrada em uma meta-análise que incluiu ensaios clínicos randomizados que compararam high-flow nasal oxygen (HFNO, terapia nasal de alto fluxo), VNI com máscara facial, VNI com capacete e oxigenoterapia padrão. ${ }^{(7)}$ Esses autores mostraram que a VNI aplicada com capacete associou-se a menor risco de intubação traqueal e morte quando comparada com as outras três opções. Os seguintes fatores podem explicar os melhores resultados da VNI com capacete: 1 . melhor tolerância com a interface do capacete minimiza as interrupções na terapia e pode aumentar sua eficácia; e 2. a interface do capacete diminui os vazamentos e pode ser mais eficaz na entrega de níveis mais elevados de PEEP, aumentando o recrutamento alveolar e a oxigenação. ${ }^{(8)}$ A interface ELMO foi bem tolerada e permitiu a aplicação de níveis de PEEP de 8 a $12 \mathrm{cmH}_{2} \mathrm{O}$, mostrando que pode ser eficaz no tratamento da IRHA. ${ }^{(4)}$ No entanto, esses resultados são preliminares e mais estudos são necessários para determinar o real papel do ELMOcpap no tratamento da IRHA.

Em pacientes com IRHA por COVID-19 em particular, a eficácia do tratamento com VNI com capacete foi demonstrada. Um ensaio clínico randomizado( ${ }^{(9)}$ que incluiu pacientes com COVID-19 e IRHA de moderada a grave $\left(\mathrm{PaO}_{2} / \mathrm{FIO}_{2}<200 \mathrm{mmHg}\right)$ mostrou que o tratamento com VNI com capacete, quando comparado com HFNO, melhorou a oxigenação, reduziu a dispneia, reduziu a taxa de intubação endotraqueal (OR $=0,41$; IC95\%: $0,18-0,89 ; p=0,03)$, e aumentou o número de dias livres de ventilação mecânica invasiva em 28 dias: mediana = 28 dias (IIQ: 13-28 dias) vs. 25 dias (IIQ: 4-28 dias); $\mathrm{p}=0,04$. Apesar desses melhores resultados, a VNI com capacete não reduziu a mortalidade em UTI ou a mortalidade hospitalar quando comparada com HFNO. ${ }^{(9)}$

Uma importante limitação da VNI em pacientes com IRHA é a taxa de mortalidade entre aqueles que falharam e foram intubados, que geralmente é maior do que aqueles que são intubados sem receber VNI previamente. $(8,10)$ Em consonância com esses achados, no estudo em questão, ${ }^{(4)}$ entre os quatro pacientes que apresentaram falha no tratamento de VNI, dois faleceram (50\%). A principal hipótese para explicar a maior mortalidade em pacientes que são intubados após receber VNI é o atraso na intubação. Esse atraso pode estar associado a eventos isquêmicos cardíacos, fadiga muscular respiratória e complicações da intubação de emergência, fatores que podem piorar os desfechos dos pacientes. ${ }^{(8,10)}$ Para reduzir as taxas de falha da VNI, estudos para identificar pacientes de alto risco e estabelecer parâmetros objetivos para indicar a intubação são necessários. 
Iniciativas inovadoras capazes de aumentar de forma efetiva e segura o tratamento de pacientes críticos são extremamente importantes e, no Brasil, ainda são poucas e raras. Portanto, o notável desenvolvimento do ELMOcpap deve ser considerado um exemplo de como enfrentar um evento adverso e catastrófico, a pandemia de COVID-19, de forma criativa e engenhosa.

\section{CONTRIBUIÇÃO DOS AUTORES}

Todos os autores participaram da redação e revisão do manuscrito, bem como da aprovação da versão final.

\section{CONFLITO DE INTERESSE}

Não declarado.

\section{REFERÊNCIAS}

1. Botta M, Tsonas AM, Pillay J, Boers LS, Algera AG, Bos LDJ, et al Ventilation management and clinical outcomes in invasively ventilated patients with COVID-19 (PRoVENT-COVID): a national, multicentre observational cohort study. Lancet Respir Med. 2021;9(2):139-148. https://doi.org/10.1016/S2213-2600(20)30459-8

2. Ranzani OT, Bastos LSL, Gelli JGM, Marchesi JF, Baiao F, Hamacher $S$, et al. Characterisation of the first 250,000 hospital admissions for COVID-19 in Brazil: a retrospective analysis of nationwide data. Lancet Respir Med. 2021;9(4):407-418. https://doi.org/10.1016/ S2213-2600(20)30560-9

3. Bravata DM, Perkins AJ, Myers LJ, Arling G, Zhang Y, Zillich AJ, et al. Association of Intensive Care Unit Patient Load and Demand With Mortality Rates in US Department of Veterans Affairs Hospitals During the COVID-19 Pandemic. JAMA Netw Open. 2021;4(1):e2034266. https://doi.org/10.1001/jamanetworkopen.2020.34266

4. Tomaz BS, Gomes GC, Lino JA, Menezes DGA, Soares JB, Furtado $V$, et al. ELMO, a new helmet interface for CPAP to treat COVID19-related acute hypoxemic respiratory failure outside the ICU: a feasibility study. J Bras Pneumol. 2022;48(1):e20210349.

5. Dar M, Swamy L, Gavin D, Theodore A. Mechanical-Ventilation Supply and Options for the COVID-19 Pandemic. Leveraging All Available Resources for a Limited Resource in a Crisis. Ann Am Thorac Soc. 2021;18(3):408-416. https://doi.org/10.1513/ AnnalsATS.202004-317CME
6. Wendel Garcia PD, Aguirre-Bermeo H, Buehler PK, Alfaro-Farias M, Yuen B, David S, Tschoellitsch T, et al. Implications of early respiratory support strategies on disease progression in critical COVID-19: a matched subanalysis of the prospective RISC-19-ICU cohort. Crit Care. 2021;25(1):175. https://doi.org/10.1186/s13054 021-03580-y

7. Ferreyro BL, Angriman F, Munshi L, Del Sorbo L, Ferguson ND, Rochwerg B, et al. Association of Noninvasive Oxygenation Strategies With All-Cause Mortality in Adults With Acute Hypoxemic Respiratory Failure: A Systematic Review and Meta-analysis. JAMA 2020;324(1):57-67. https://doi.org/10.1001/jama.2020.9524

8. Nava $\mathrm{S}$, Hill N. Non-invasive ventilation in acute respiratory failure. Lancet. 2009;374(9685):250-259. https://doi.org/10.1016/S0140 6736(09)60496-7

9. Grieco DL, Menga LS, Cesarano M, Rosa T, Spadaro S, Bitondo MM, et al. Effect of Helmet Noninvasive Ventilation vs High-Flow Nasal Oxygen on Days Free of Respiratory Support in Patients With COVID-19 and Moderate to Severe Hypoxemic Respiratory Failure: The HENIVOT Randomized Clinical Trial. JAMA. 2021;325(17):1731 1743. https://doi.org/10.1001/jama.2021.4682

10. Esteban A, Frutos-Vivar F, Ferguson ND, Arabi Y, Apezteguia C Gonzalez $M$, et al. Noninvasive positive-pressure ventilation for respiratory failure after extubation. N Engl J Med. 2004;350(24):2452 2460. https://doi.org/10.1056/NEJMoa032736 